\title{
Educação, comunicação e mobilização social, estratégias de promoção da saúde no monitoramento de vetores em assentamentos rurais: possibilidades e desafios
}

\author{
Education, communication and social mobilization, health promotion strategies in vector \\ monitoring in rural settlements: possibilities and challenges
}

Educación, comunicación y movilización social, estrategias de promoción de la salud en el monitoreo de vectores en asentamientos rurales: posibilidades y desafíos

\author{
João Carlos de Oliveira ${ }^{1}$ \\ Vinícius Bonassi ${ }^{2}$ \\ Guilherme Macedo de Melo ${ }^{3}$
}

RESUMO: Este trabalho é resultado de uma Roda de Conversa, realizada durante a V Jornada Universitária em Defesa da Reforma Agrária, fruto das parcerias, no monitoramento de vetores, entre a Escola Técnica de Saúde da Universidade Federal de Uberlândia, o Instituto Federal de Educação, Ciência e Tecnologia - Campus Uberlândia e Associação de Recicladores e Catadores Autônomos. A degradação ambiental tem disseminado arbovírus, entre eles os Aedes (Aegypti e albopictus) e o Culex e suas arboviroses. Nas áreas urbanas, temos a Dengue, Chikungunya, Febre do Nilo Ocidental, Zika, Rocio e Mayaro; em ambientes rurais (e até urbanos), e alguns casos silvestres, temos os flebotomíneos, popularmente chamados de "mosquito palha" ou "cangalhinha" responsáveis pela Leishmaniose Visceral e/ou Leishmaniose Tegumentar e os Haemagogus e Sabethes, responsáveis pela Febre Amarela Silvestre. A Roda de Conversa ocorreu na concepção e construção dialógica sobre "O que faço, o que sei e o que precisamos saber de saúde ambiental em relação aos vetores", considerando os estilos e modos de vidas das pessoas. Por isso, a importância de um processo educativo, da comunicação e mobilização social como forma de estabelecer ambientes saudáveis enquanto estratégias da promoção da saúde.

Palavras-chave: Mobilização social. Comunicação. Monitoramento de vetores. Assentamentos rurais.

\begin{abstract}
This work is the result of the Conversation Circle held during the V University Day in Defense of Land Reform, which has a direct relationship of partnerships in vector monitoring between the Federal University of Uberlândia Technical School of Health, Federal Institute of Education, Science and Technology - Uberlândia Campus and Association of Autonomous Recyclers and Pickers. Environmental degradation has spread arboviruses, including Aedes (Aegypti and albopictus) and Culex and its arboviruses. In urban areas Dengue, Chikungunya, West Nile Fever, Zika, Rocio and Mayaro; in rural (and even urban) environments, and in some wild cases, we have sand flies, popularly called "straw mosquito" or "cangalhinha" responsible for Visceral Leishmaniasis and/or Tegumentary Leishmaniasis; Haemagogus and Sabethes are responsible for the Yellow Wild Fever. The Conversation Circle occurred in the conception and dialogical construction about "What I do, what I know and what we need to know about environmental health in relation to vectors", considering

1 Doutor em Geografia pela Universidade Federal de Uberlândia, Minas Gerais, Brasil, com período sanduíche na Universidade Federal do Amazonas, Brasil; professor na Escola Técnica de Saúde da Universidade Federal de Uberlândia, Minas Gerais, Brasil (oliveirajotaufuestes@gmail.com).

${ }^{2}$ Graduando em Gestão em Saúde Ambiental no Instituto de Geografia da Universidade Federal de Uberlândia, Minas Gerais, Brasil (vini-bonassi1@ hotmail.com).

${ }^{3}$ Graduando em Gestão em Saúde Ambiental no Instituto de Geografia da Universidade Federal de Uberlândia, Minas Gerais, Brasil (gmacedo970@gmail.com).
\end{abstract}


people's styles and ways of life. Therefore, the importance of an educational process, communication and social mobilization, as a ways to establish healthy environments, as strategies of health promotion.

Keywords: Social mobilization. Communication. Vector monitoring. Rural settlements.

RESUMEN: Este trabajo es el resultado de una Rueda de Conversación realizada en la V Jornada Universitaria en Defensa de la Reforma Agraria, el resultado de alianzas en el monitoreo de vectores entre la Escuela Técnica de Salud de la Universidad Federal de Uberlândia, el Instituto Federal de Educación, Ciencia y Tecnología del Campus Uberlândia y la Asociación de Recicladores y Recolectores Autónomos. La degradación ambiental ha extendido los arbovirus, entre los Aedes (Aegypti y albopictus) y Culex y sus arbovirosis. En zonas urbanas el Dengue, Chikungunya, Fiebre del Nilo Occidental, Zika, Rocío y Mayaro. En ambientes rurales (y hasta urbanos), y en algunos casos salvajes, tenemos los flebotominos, responsables de la Leishmaniasis Visceral y/o Cutánea. Por otra parte los Haemagogus y Sabethes son responsables de la fiebre amarilla salvaje. La Rueda de Conversación estuvo basada en la concepción y construcción del dialogo sobre "lo que hago, lo que sé y lo que necesitamos saber sobre la salud ambiental en relación con los vectores", considerando los estilos y formas de vida de las personas. Por esta razón, la importancia de un proceso educativo, de comunicación y movilización social como una forma de establecer ambientes saludables, como estrategias de promoción de la salud.

Palabras clave: Movilización social. Comunicación. Monitoreo de vectores. Asentamientos rurales.

\section{INTRODUÇÃO}

Este trabalho é resultado de uma Roda de Conversa realizada durante a V Jornada Universitária em Defesa da Reforma Agrária (JURA), que aconteceu na Universidade Federal de Uberlândia (UFU), concomitante ao $9^{\circ}$ Simpósio Internacional: O Estado e as Políticas Educacionais no Tempo Presente e a VII Feira Regional de Economia Popular Solidária. A Jornada, que contou com diferentes segmentos da sociedade, teve como objetivo socializar e debater experiências sobre a importância da reforma agrária popular e sua relação com outros grupos da sociedade.

Para a condução da Roda de Conversa, valemo-nos de estudos e pesquisas que vêm sendo desenvolvidos por meio de projetos ${ }^{4}$, desde 2013, em parceria com a Escola Técnica de Saúde (ESTES), o Instituto Federal de Educação, Ciência e Tecnologia do Triângulo Mineiro

\footnotetext{
${ }^{4}$ Projetos aprovados por Editais: 1) Financiado pela Fundação de Amparo à Pesquisa do Estado de Minas Gerais (FAPEMIG), Edital no 07/2013 - Projeto de extensão em interface com pesquisa (CHE-APQ-02914-13); 2) UFU - "Mobilização social e redes locais em microterritórios no monitoramento de vetores, utilizando ovitrampas, enquanto estratégias de promoção da saúde: possibilidades e desafios"; 3) IFTM -"Mobilização comunitária e contribuições de agentes ambientais em microterritórios no monitoramento de vetores: estratégias de promoção da saúde com escolas".
} 
(IFTM) - Campus Uberlândia, e a Associação de Recicladores e Catadores Autônomos $\left(\mathrm{ARCA}^{5}\right)$, levando em consideração os trabalhos de Oliveira (2006; 2012), no monitoramento, por meio de ovitrampas, de arbovírus (vetores/mosquitos, em especial (Aedes e Culex ${ }^{6}$ ), com as suas potenciais arboviroses, ou também denominadas doenças negligenciadas ou reemergentes.

Nas áreas urbanas, temos a Dengue, Chikungunya, Febre Amarela, Febre do Nilo Ocidental e Zika, sendo esta última um motivo de preocupação por sua associação com a microcefalia em recém-nascidos. Já em ambientes rurais, e alguns casos silvestres, temos os flebotomíneos, popularmente chamados de "mosquito palha" ou "cangalhinha", responsáveis pela Leishmaniose Visceral e/ou Leishmaniose Tegumentar; e os Haemagogus e Sabethes, responsáveis pela Febre Amarela Silvestre.

Para este trabalho, abordaremos a importância da educação, comunicação e mobilização social como estratégias de promoção da saúde em assentamentos rurais, exatamente porque, em vários momentos, são os assentados que estão expostos a riscos, ficando vulneráveis a algumas dessas arboviroses, pois, na maioria das vezes, tudo é mosquito e há uma naturalização dos vetores e das doenças.

As Rodas de Conversas realizadas durante o evento ocorreram de forma coletiva. Nelas, foram compartilhados informações e conhecimentos a partir da realidade de cada participante. Assim, as informações foram socializadas no intuito de refletir e comunicar as experiências sobre o tema sugerido, como forma de "desnaturalizar" sobre as necessidades dos cuidados ambientais em relação aos vetores. Até porque esses "cuidados" estão relacionados aos Determinantes Sociais de Saúde (DSS). De acordo com Buss e Pellegrini Filho (2007), os $\mathrm{DSS}^{7}$ "são os fatores sociais, econômicos, culturais, étnicos/raciais, psicológicos e

\footnotetext{
${ }^{5}$ A Arca faz parte das Organizações Produtivas Solidárias (OPS) e é uma das associações incubadas pelo Centro de Incubação de Empreendimentos Populares Solidários (Cieps), cuja missão é promover a economia popular e solidária, assessorando coletivos populares que gerem trabalho e renda dentro dos princípios da economia solidária por meio da indissociabilidade do ensino, pesquisa e extensão.

${ }^{6}$ Este vetor vem despertando interesse para diferentes estudos, a tal ponto que um grupo de cientistas do Departamento Médico da Universidade do Texas, em Galveston (UTMB) sequenciou o genoma de um dos mosquitos mais domésticos do ambiente tropical (http://noticias.ambientebrasil.com.br/clipping/2010/10/01/61059-cientistas-sequenciam-o-genoma-domosquito-domestico-tropical.html).

${ }^{7}$ A comissão homônima da Organização Mundial da Saúde (OMS) adota uma definição mais curta, segundo a qual os DSS são as condições sociais em que as pessoas vivem e trabalham. Nancy Krieger (2001) introduz um elemento de intervenção, ao defini-los como os fatores e mecanismos através dos quais as condições sociais afetam a saúde e que potencialmente podem ser alterados através de ações baseadas em informação. Tarlov (1996) propõe, finalmente, uma definição bastante sintética, ao entendê-los como as características sociais dentro
} 
comportamentais que influenciam a ocorrência de problemas de saúde e seus fatores de risco na população".

É importante destacar que outros temas afloraram nas falas e nas reflexões de interesses dos/as participantes, desde agroecologia, educação ambiental, economia solidária e saúde do trabalhador. Assim, pudemos estabelecer correlações diretas entre esses temas trazidos e o que por ora apresentávamos.

Como forma de aproximar a proposta de nosso trabalho ao contexto dos assentamentos rurais, apresentamos uma letra de música para comunicar a importância do monitoramento de vetores no contexto dos assentamentos rurais:

\section{A caneta e a enxada ${ }^{8}$}

Certa vez uma caneta foi passeá lá no sertão Encontrou-se com uma enxada, fazendo uma prantação. A enxada muito humirde, foi lhe fazer saudação, Mas a caneta soberba não quis pegar na sua mão. E ainda por desaforo the passou uma repreensão.

Disse a caneta pra enxada não vem perto de mim, não Você está suja de terra, de terra suja do chão Sabe com quem está falando, veja sua posição E não se esqueça a distância da nossa separação.

Eu sou a caneta dourada que escreve nos tabelião Eu escrevo pros governos a lei da constituição Escrevi em papel de linho, pros ricaços e pros barão Só ando na mão dos mestres, dos homens de posição.

A enxada respondeu: de fato eu vivo no chão, Pra poder dar o que comer e vestir o seu patrão Eu vim no mundo primeiro, quase no tempo de Adão Se não fosse o meu sustento ninguém tinha instrução.

Vai-te caneta orgulhosa, vergonha da geração A tua alta nobreza não passa de pretensão Você diz que escreve tudo, tem uma coisa que não É a palavra bonita que se chama educação!

Essa letra nos remete a alguns contextos. Um deles diz sobre a relação entre quem tem educação formal (caneta) e quem não tem (enxada). Outro, sobre a Divisão Social do Trabalho (DST) que, em termos genéricos, refere-se às diferentes formas como os seres

das quais a vida transcorre (Fonte: http://renastonline.ensp.fiocruz.br/temas/determinantes-sociais-saude. Data de acesso: outubro de 2019).

${ }^{8}$ Composição de Capitão Barduíno e Teddy Vieira; interpretação de Zico e Zeca. Para maiores informações: https://www.letras.mus.br/zico-e-zeca/235558/. 
humanos - para viverem, produzirem e reproduzirem a/na vida - especializam e espacializam, de forma desigual, em forma de mercadorias, ou não, a sua produção, a partir da força de trabalho empregada. Esse processo produtivo e reprodutivista não nos permite romper com os modelos de educação, aqui em especial dessa que muito mais adestra, domestica e reproduz um modelo de (re)produção do mundo do trabalho, de interesse de pequenos grupos donos do capital e dos meios de produção. Se assim, não concordamos e desejamos romper com esse modelo de exploração da mão de obra do trabalhador, e os diálogos com diferentes segmentos da sociedade, de forma mais horizontal, poderá romper com esse modelo.

Nesse sentido, aludimos ao método criado por Tião Rocha, fundador do Centro Popular de Cultura e Desenvolvimento (CPCD), Belo Horizonte/MG, conhecido como "Pedagogia de Roda". O método configura-se como um espaço horizontal em que todos colocam suas "visões de mundo" com o intuito de aprender e ensinar.

E foi mais ou menos o que aconteceu com a Roda de Conversa conduzida por nós no V JURA junto aos trabalhadores/as do/no campo. Corroboramos o pensamento de Thiollent (1980), que vê o relacionamento entre as pessoas como uma comunicação de informações num determinado espaço sociopolítico, que pode variar de acordo com as populações, os problemas investigados, sua relação com o poder, os grupos e instituições de pesquisa, os interesses que estão em jogo etc.

Assim, entendemos ser necessário mobilizar as pessoas. Mas como? Por meio da (in)formação, da mobilização e da educação em saúde, em específico, em relação aos cuidados ambientais, sob a perspectiva da "Educomunicação"9.

Para podermos pensar e vivenciar a Educação Popular em Saúde e a Educomunicação apoiamo-nos em Valla e Stotz $(1993 ; 1994)$, que compreendem que a educação popular é um fator de transformação da sociedade, mas, ao mesmo tempo, desvinculam as "intenções e desejos" dos agentes de educação popular de uma obrigatória transformação social. Ainda segundo os autores, não devemos achar que a educação popular, por si mesma, traz necessariamente em seu bojo as sementes da transformação social. Trata-se de uma questão que inclui variáveis como a conjuntura política e econômica, a organização das pessoas em questão e a sensibilidade política e cultural dos agentes. Porém, de qualquer forma, a

\footnotetext{
${ }^{9}$ O termo foi empregado pela primeira vez pelo filósofo da educação Mario Kaplun para referir-se à
} convergência entre as áreas de comunicação e educação (NOGUEIRA; TONUS, 2010). 
formação e a comunicação só terão eficiência e/ou eficácia se tivermos uma significativa mobilização, que, de acordo com Oliveira (2012), não nasce espontaneamente, é preciso que as pessoas sintam-se pertencentes e se identifiquem com o que está sendo proposto, principalmente ao responder demandas emergentes, aqui, neste caso, epidemias de arbovírus, que muitas vezes reflete estilos e modos de vidas, necessitando novos saberes e fazeres.

Diante dessas proposições, senão provocações, apresentamos os resultados da Roda de Conversa, realizada durante a V JURA, cujo foco não foi os mosquitos, mas a mobilização social, por meio das ovitrampas (Figuras 1 e 2).

Figuras 1 e 2 - Modelos de ovitrampas
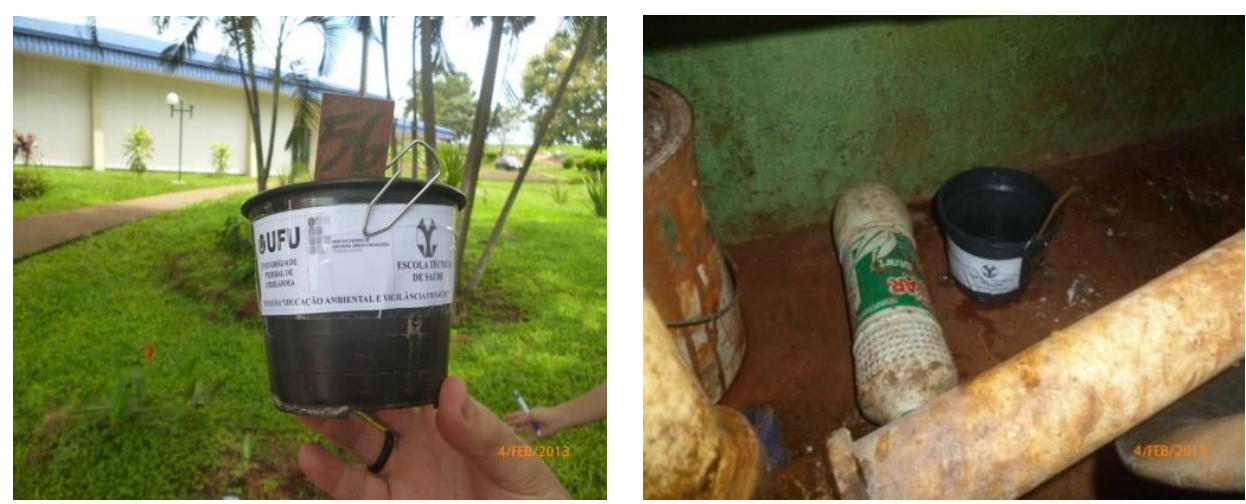

Fonte: Acervo pessoal (2018).

De acordo com BRASIL (2001), as ovitrampas são depósitos de plástico preto com capacidade de $500 \mathrm{ml}$ de água e uma palheta de Eucatex, em que as fêmeas depositam os ovos. A inspeção é semanal, quando então as palhetas são recolhidas e encaminhadas para laboratório e substituídas por outras. Constituem método sensível e econômico na detecção precoce da presença e de infestações de vetores, em diferentes períodos sazonais e lugares.

$\mathrm{Na}$ parte rugosa das palhetas, onde as fêmeas realizam a oviposição (Figuras 3 e 4), com o auxílio de lupa estereomicroscópica, é possível identificar e quantificar os ovos - viáveis, eclodidos e danificados - de cada vetor, bem como a espacialidade dele e, aqui, uma "maior" visibilidade dos riscos e das vulnerabilidades ambientais.

Figuras 3 e 4 - Palhetas com ovos viáveis e eclodidos 

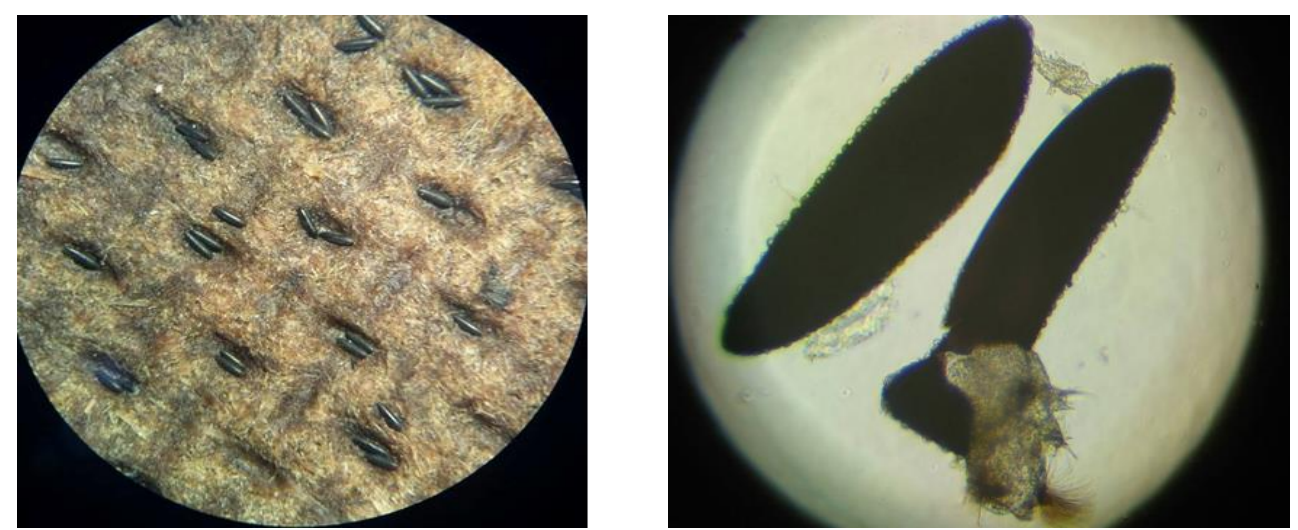

Fonte: Acervo pessoal (2018).

Logicamente que as ovitrampas e a presença de ovos deram visibilidade de um (des)conhecimento e de uma "naturalização" muito comum de que tudo é mosquito e eles, muitas vezes, são de importância para a Saúde Pública. Em áreas urbanas, os arbovírus Aedes (aegypti e albopictus) e Culex causam, principalmente, Dengue, Febre Chikungunya, Febre do Nilo Ocidental (FNO), Mayaro, Rocio e Febre Zika. Em ambientes rurais, e alguns casos silvestres, temos o "mosquito palha" ou "cangalhinha", responsável pela Leishmaniose Visceral e/ou Leishmaniose Tegumentar. Já os Haemagogus e Sabethes são responsáveis pela Febre Amarela Silvestre.

O Aedes aegypti é o principal vetor responsável pela dengue no Brasil, também é o mais combatido, para o qual se faz mais propaganda e uso de recursos públicos (URBINATTI; NATAL, 2009). Há um paradoxo aqui. De um lado, o fracasso do modelo atual de Vigilância, em função dos inúmeros casos de arboviroses, impactando a saúde das pessoas, causando mortes, afastamentos laborais, escolares, promovendo mudanças de rotinas hospitalares. De outro, com custos sociais, de forma coletiva, quase todos pagam as contas ${ }^{10}$.

Também merece atenção outros vetores, como o Aedes albopictus e o Culex, que de acordo com Urbinatti e Natal (2009, p. 279),

\footnotetext{
${ }^{10}$ Em janeiro de 2017, o Ministério da Saúde repassou R \$ 91,2 milhões aos municípios de todos os estados, referentes à primeira parcela de um total de $\mathrm{R} \$ 152$ milhões destinados ao combate ao Aedes. Já o envio da segunda parcela foi condicionado ao cumprimento de alguns critérios, especialmente realização do Levantamento Rápido de Índice de Infestação por Aedes aegypti (LIRAa), no caso de cidades com mais de dois mil imóveis. Os municípios com menos de dois mil imóveis devem fazer o Levantamento de Índice Amostral (LIA) e o monitoramento por ovitrampa ou larvitrampa nas cidades sem infestação do mosquito. O Ministério da Saúde liberou, no mês de setembro, R\$ 30,4 milhões, referentes à segunda parcela de recurso adicional para o combate ao mosquito Aedes aegypti. Ao todo, 3.148 municípios em 20 estados e o Distrito Federal foram beneficiados porque cumpriram critérios para intensificar as medidas de prevenção e combate ao mosquito transmissor da Dengue, Zika e Chikungunya (http://combateaedes.saude.gov.br/pt/noticias/910-ministerio-dasaude-repassa-r-30-4-milhoes-de-recurso-extra-para-combate-ao-aedes).
} 
Tem uma correlação com o Vírus do Nilo Ocidental (VNO) e outras arboviroses, junto com o Culex, provocam riscos de encefalite e outras viroses. O Culex é Culicíneos - do gênero Culex, que no Brasil destaca-se a espécie Culex quinquefasciatus por transmitir a Wuchereria bancrofti, agente da Filariose em cidades do norte e nordeste. Essa espécie, sinantrópica, de elevada antropofilia, devido à sua atividade hematofágica está geralmente associada a coleções aquáticas estagnadas e poluídas por efluentes de esgoto domésticos ou industriais.

Lembrando que a maioria dos casos ocorre durante o verão, especialmente nos meses de maior precipitação atmosférica. Mas não se pode imputar ao clima a causa das doenças, nem mesmo aos mosquitos (arbovírus), como aparece nas campanhas veiculadas nos meios de comunicação. Há um conjunto de Determinantes Sociais de Saúde (DSS) que nos indicam certos riscos e vulnerabilidades das pessoas.

Preocupado com cenários de epidemias de arboviroses, o Ministério da Saúde, em 2009, elabora as Diretrizes Nacionais para Prevenção e Controle da Dengue (DNPCD) ${ }^{11}$, alicerçadas em quatro componentes: 1) Controle Vetorial, 2) Vigilância Epidemiológica, 3) Assistência e 4) Comunicação e Mobilização.

$\mathrm{Na}$ verdade, todo processo saúde-doença é multicausal. Na maioria das vezes, as medidas adotadas são higienistas/sanitaristas, de concepção do modelo biomédico hospitalocêntrico/flexneriano. Sobre esse modelo existem vários estudos, aqui nos aportamos aos de Pagliosa e Ros (2008) e Almeida Filho (2010).

Para Pagliosa e Ros (2008, p. 496),

Mesmo que consideremos muito importantes suas contribuições para a educação médica, a ênfase no modelo biomédico, centrado na doença e no hospital, conduziu os programas educacionais médicos a uma visão reducionista. Ao adotar o modelo de saúde-doença unicausal, biologicista, reserva pequeno espaço para as dimensões social, psicológica e econômica da saúde e para a inclusão do amplo espectro da saúde, que vai muito além da medicina e seus médicos, que evidencia um descompromisso com a realidade e as necessidades da população.

Almeida Filho (2010, p. 2.239-2.240), nos lembra que,

Aparentemente, o modelo biomédico de educação médica foi em princípio delineado por Eugênio Vilaça Mendes, odontólogo, consultor da OPAS, membro atuante do Departamento de Medicina Preventiva da Universidade

\footnotetext{
${ }^{11}$ http://bvsms.saude.gov.br/bvs/publicacoes/diretrizes_nacionais_prevencao_controle_dengue.pdf.
} 
Federal de Minas Gerais (UFMG), foco nacional do movimento da Integração Docente-Assistencial e das propostas de reforma curricular promovidas pela Associação Brasileira de Educação Médica (ABEM). [...]. Num par de textos, complementados posteriormente por um livro de síntese doutrinária intitulado Uma Agenda para a Saúde (1996), Mendes explicita os elementos estruturais do modelo biomédico suposto como flexneriano: mecanicismo, biologismo, individualismo, especialização, exclusão de práticas alternativas, tecnificação do cuidado à saúde, ênfase na prática curativa.

Diante disso, o Ministério da Saúde institui a Política Nacional de Educação Popular em Saúde no âmbito do Sistema Único de Saúde (PNEPS-SUS), por meio da Portaria $\mathrm{n}^{\circ}$ 2.761, de 19 de novembro de 2013 (BRASIL, 2013), baseada em quatro eixos estratégicos: Participação, controle social e gestão participativa; Formação, comunicação e produção de conhecimento; Cuidado em saúde; Intersetorialidade e diálogos multiculturais.

Entendemos que os participantes da Roda de Conversa protagonizaram o proposto pela Política Nacional de Educação Popular em Saúde, em especial pelos diferentes diálogos e proposições, vislumbrando possibilidade de parcerias, por exemplo, no monitoramento de vetores em algum assentamento rural. Esse estreitamento entre pesquisadores e participantes, nos faz lembrar do que disse Minayo (2011, p. 18) sobre a Conferência Mundial sobre Ciência, ocorrida em Budapeste, em 1999:

a) a ciência deve estar a serviço de toda a humanidade; b) a ciência deve contribuir para o conhecimento mais profundo da natureza e da sociedade; c) a ciência deve contribuir para a qualidade de vida e para criar um ambiente saudável para as gerações presentes e futuras.

Assim, entendemos e reconhecemos a importância dos estudos e das pesquisas, mas dentro do contexto que se aproximem das pessoas, beneficiárias das informações e dos conhecimentos produzidos. A conhecimento é de interesse coletivo.

$\mathrm{Na}$ verdade o aparecimento de doenças, a partir do ambiente poluído e/ou contaminado, muitas vezes é de responsabilidade antrópica, o que nos trouxe preocupações com a (in)salubridade dos ambientes na produção das doenças.

O que por hora Foucault (1979, p. 93) dizia que:

Salubridade não é a mesma coisa que saúde, e sim o estado das coisas, do meio e seus elementos constitutivos, que permitem a melhor saúde possível. Salubridade é a base material e social capaz de assegurar a melhor saúde 
possível dos indivíduos. E é correlativamente a ela que aparece a noção de higiene pública, técnica de controle e de modificação dos elementos materiais do meio que são suscetíveis de favorecer ou, ao contrário, prejudicar a saúde. Salubridade e insalubridade são o estado das coisas e do meio enquanto afetam a saúde; a higiene pública - no século XIX, a noção essencial da medicina social francesa - é o controle político-científico deste meio.

$\mathrm{Na}$ verdade esse "controle político-científico" ocorre com "a medicalização da cidade, a medicina urbana e a medicina social” que, de acordo com Foucault (1979, p. 96),

Tinham por função: $1^{\circ}$ ) Controle da vacinação, obrigando os diferentes elementos da população a se vacinarem. $2^{\circ}$ ) Organização do registro das epidemias e doenças capazes de se tornarem epidêmicas, obrigando as pessoas à declaração de doenças perigosas. $3^{\circ}$ ) Localização de lugares insalubres e eventual destruição desses focos de insalubridade.

Quando da abordagem "medicalização da cidade", chamamos atenção para determinados lugares, por exemplo, a "casa e a rua" das cidades, muitas vezes implantadas nos contextos dos "assentamentos rurais", enquanto espaços higienizados e/ou responsáveis, ou não, na maioria das vezes por conter criadouros. Assim, como fica essa concepção de higienização?

Para Oliveira (1998), a casa é o abrigo, o santuário, o bem, o bom, o belo, o sagrado, o significado dos bons fluídos, dos bons costumes. A rua é o inverso, o feio, o lugar do não lugar, o inferno, o espaço do perverso que ensina as más lições, o lugar da perdição, do abandono, da dispersão, da sujeira, da imundície, do submundo. Já o "rural/campo" é o belo, a pureza, a presença da natureza, a liberdade. Mas há um contrassenso dos riscos a que as pessoas estão expostas, muitas delas não têm a dimensão dos perigos.

A presença dos arbovírus está associada a um conjunto de condições ambientais. De um lado, a dinâmica natural, em especial umidade e temperatura, não mais tão determinantes nesses últimos anos; do outro, a dinâmica social, muito mais pelos estilos e modos de vidas das pessoas, e os arbovírus estão cada vez mais presentes dentro das nossas moradias. Queremos com isso dizer que a distribuição espacial dos vetores e das doenças em um determinado território tem relação com alguns aspectos geográficos, predominantemente os climáticos, mas também com outros fatores: físicos (relevo, solos, hidrografia etc.), sociais (distribuição e densidade da população, estilos, modos e padrão de vida, costumes religiosos e superstições, meios de comunicação) e biológicos (tipos de vidas vegetal e animal, parasitismos, doenças predominantes, grupo sanguíneo da população, entre outras). 
As atividades, por si só, não resolvem certas contradições relacionadas aos arbovírus, mas apontam novos rumos. Fernández (2001a; 2001b), em "O saber em jogo e a autoria do pensamento", discorre sobre a etimologia de "intervir (vir entre)" e "interferir (ferir entre)". Ainda que, às vezes, necessitamos interferir, tenderemos a que nossa intervenção seja da ordem de uma 'inter-versão’ (incluir outra versão), sem anular as outras possibilidades.

Por isso, retomamos as ideias sobre Promoção da Saúde, complementado por Sarlet e Figueiredo (2009). Este autores nos dizem que a Constituição Brasileira de 1988, já vislumbrava uma mudança paradigmática na conceituação do tema direito à saúde, trazendo uma abordagem mais abrangente, conforme a Organização Mundial da Saúde (OMS). Para os referidos autores, a OMS propõe algo para além de uma noção eminentemente curativa, sendo a nova proposta compreendida entre as dimensões preventivas e promocionais na tutela dos direitos fundamentais dos indivíduos, ou seja, os Direitos Humanos.

De acordo com Sarlet e Figueiredo (2009), é mais apropriado falar-se não simplesmente em direito à saúde, mas no direito à proteção e à promoção da saúde, inclusive como "imagemhorizonte" a ser perseguida. Como bem colocam os autores, as expressões "redução do risco de doença" e "proteção" direcionam-se à temática "saúde preventiva", que é em tese a realização de ações e políticas de saúde que tenham como objetivo evitar o surgimento da doença.

Dessa forma, as (in)formações, as mobilizações e a educação em saúde nos monitoramentos de vetores, levados para as rodas de conversa, podem proporcionar possibilidades de novos saberes e fazeres de formas distintas para fazer diferenças, em relação aos cuidados com a saúde ambiental, enquanto estratégia de promoção da saúde.

\section{CONSIDERAÇÕES FINAIS}

No Brasil, ainda que o verão e os vetores sejam fatores determinantes da ocorrência das arboviroses, não se pode imputar os mesmos como únicos responsáveis pelas doenças, como evidenciadas massivamente em campanhas, pois o processo ambiente-saúde-doença é multicausal.

As estratégias de monitoramento de vetores foram realizadas a partir das atividades: (re)conhecimento das realidades vividas pelos sujeitos; educação e saúde; mobilização social e práticas de vigilância ambiental e epidemiológica. 
Os resultados obtidos com o presente estudo no monitoramento de vetores e nos resultados da Roda de Conversa apresentam grande relevância para a Saúde Pública e Epidemiologia, como possibilidade de formação de profissionais da área da educação e de implantação em outras comunidades, pelo baixo custo e boa eficiência, enquanto estratégia de vigilância ambiental, que dada a efetividade (experiências colaborativas), sugere-se a replicação dessa experiência exitosa com diversos segmentos, em especial aos assentados rurais da região de Uberlândia, e quiçá do Brasil.

\section{REFERÊNCIAS}

ALMEIDA FILHO, N. Reconhecer Flexner: inquérito sobre produção de mitos na educação médica no Brasil contemporâneo. Cadernos de Saúde Pública, Rio de Janeiro, v. 26, n. 12, p. 2.234-2.249, dez. 2010. Doi: 10.1590/S0102-311X2010001200003.

BUSS, P. M.; PELLEGRINI FILHO, A. A saúde e seus determinantes sociais. Physis [online], Rio de Janeiro, v. 17, n. 1, p. 77-93, abr. 2007. Doi: 10.1590/S010373312007000100006.

BRASIL. Ministério da Saúde. Fundação Nacional de Saúde. Instruções para pessoal de combate ao vetor: manual de normas técnicas. Brasília: Ministério da Saúde, 2001.

BRASIL. Ministério da Saúde. Política Nacional de Educação Popular em Saúde no âmbito do Sistema Único de Saúde (PNEPS-SUS). Brasília: Ministério da Saúde, 2013.

BRASIL. Ministério da Saúde. Secretaria de Vigilância em Saúde, Departamento de Vigilância Epidemiológica. Diretrizes Nacionais para a Prevenção e Controle da Dengue. Brasília: Ministério da Saúde, 2009.

FERNÁNDEZ, A. Os idiomas do aprendente: análise das modalidades ensinantes com família, escolas e meios de comunicação. Porto Alegre: Artmed, 2001.

FERNÁNDEZ, A. O saber em jogo: a psicopedagogia propociando autorias de pensamento comunicação. Porto Alegre: Artmed, 2001.

FOUCAULT, M. Micro-física do poder. SP: Graal, 1979.

MINAYO, M. C. S. Indivíduo e sociedade: pesquisadores debatem a dimensão social das questões da saúde. 2011. Disponível em:

http://www.ensp.fiocruz.br/radis/sites/default/files/107/pdf/radis-107.pdf. Acesso: 20 jan. 2020.

OLIVEIRA, J. C. A casa e a rua: frutos do trabalho social ou local de dominação? Boletim Goiano de Geografia, Goiânia, v. 18, n. 1, p. 47-62, 1998. Doi:

doi.org/10.5216/bgg.v18i1.4288. 
OLIVEIRA, J. C. Manejo integrado para controle do Aedes e prevenção contra a dengue no Distrito de Martinésia, Uberlândia (MG). 2006. 58 f. Dissertação (Mestrado em Geografia) - Programa de Pós-Graduação em Geografia, Universidade Federal de Uberlândia, Uberlândia, 2006. Disponível <www.ig.ufu.br>. Acesso: maio de 2006.

OLIVEIRA, J. C. Mobilização comunitária como estratégia da promoção da saúde no controle dos Aedes (aegypti e albopictus) e prevenção do dengue no Distrito de Martinésia, Uberlândia (MG). 2012. 273. Tese (Doutorado em Geografia) - Programa de Pós-Graduação em Geografia, Universidade Federal de Uberlândia, Uberlândia, 2012. Disponível < http://www.ppgeo.ig.ufu.br/teses>. Acesso: março de 2012.

PAGLIOSA, F. L.; ROS, M. A. O Relatório Flexner: para o bem e para o mal. Revista Brasileira de Educação Médica, Rio de Janeiro, v. 32, n. 4, p. 492-499, out./dez. 2008. Doi: 10.1590/S0100-55022008000400012.

ROCHA, S. Educador é aquele que aprende. [Entrevista concedida a] Marcelo Ilha. Construir notícias, ed. 55, nov./dez. 2010. Disponível em:

http://www.construirnoticias.com.br/educador-e-aquele-que-aprende/. Acesso em: 20 jan. 2020.

SARLET, I. W.; FIGUEIREDO, M. F. Algumas considerações sobre o direito fundamental à proteção e promoção da saúde aos 20 anos da Constituição Federal de 1988. 2009. p. 1-35. Disponível em:

http://www.stf.jus.br/arquivo/cms/processoAudienciaPublicaSaude/anexo/O_direito_a_saude _nos_20_anos_da_CF_coletanea_TAnia_10_04_09.pdf. Acesso: 23 janeiro de 2020.

THIOLLENT, M. Crítica metodológica, investigação social e enquete operária. São Paulo: Polis, 1980.

URBINATTI, P. R.; NATAL, D. Artrópodes de importância em saúde pública. In: GIATTI, Leandro (org.). Fundamentos de saúde ambiental. Manaus: Editora da UFAM, 2009. p. 257-292.

VALLA, V.; STOTZ, E. Participação popular, educação e saúde: teoria e prática. Rio de Janeiro: Relume Dumará, 1993.

VALLA, V.; STOTZ, E. Educação, saúde e cidadania. Petrópolis: Vozes, 1994. 